TP Periodica Polytechnica Electrical Engineering and Computer Science

60(4), pp. 200-205, 2016

DOI: 10.3311/PPee.9676

Creative Commons Attribution (1)

RESEARCH ARTICLE

\section{LTCC Based Planar Inductive Proximity Sensor Design}

\author{
Samuel Zuk ${ }^{1 *}$, Alena Pietrikova ${ }^{1}$, Igor Vehec ${ }^{1}$
}

Received 29 June 2016; accepted after revision 22 August 2016

\begin{abstract}
This paper is focused on design and realization of planar inductive sensor system used for proximity sensing based on Low Temperature Co-fired Ceramics (LTCC). Planar coil consisting of six layers was designed, produced and measured. Electronic system for driving coil and processing measured values was developed and realized. Proximity measurement of various metal materials was realized and results were processed and evaluated. Measurements has shown that six-layers LTCC structure with implemented coils in cooperation with developed driving electronic is appropriate solution for design and construction of planar proximity sensor. It had been developed sensitive inductive sensor for up to 7 millimeters distance proximity measurements.
\end{abstract}

\section{Keywords \\ planar inductive proximity sensor, planar inductor, LTCC technology}

\section{Introduction}

Actual trends in electronics industry give demanding requirements on sensors used for process and devices control. Depending of sensing application, immunity against environment impact, reliability and long life cycle, robustness, small physical dimensions, low energy consumption and low manufacturing price are the main requirements for modern sensors and sensing systems. Inductive sensors meet these requirements. Event counting (rotary speed detection, flow meters), simple buttons for industrial keypads, industry switches, metal detection, linear position detection, angle detection, engine tests (valve position), roller gap process control (thickness, shape, texture), quality inspection (deformation, cracks) or vibration sensing are only some of the possible applications of inductive sensors. Because of any mechanical parts, inductive sensors are ideal option for proximity detection.

Harsh environment, low available space and the need of inductive proximity sensing are the main reasons to choose an alternative to standard inductive sensors. Low Temperature Co-fired Ceramic (LTCC) system offers very good alternative for producing planar inductive proximity sensor. LTCC is technology that allows creating of multilayer modules by stacking single ceramic sheets together. Glass-ceramic composite material of LTCC tapes usually consists of $\mathrm{Al}_{2} \mathrm{O}_{3}, \mathrm{SiO}_{2}$ and organic materials. Sheet of LTCC can be shaped and bended before firing process. LTCC tape processing is based on screen printing, laminating and firing process. Applying of conductive, dielectric and resistive pastes LTCC technology allows to integrate resistors, capacitors and inductors. Resistance against high temperatures and negative impact of given environment $[1,2]$, ability to produce planar conductors in tenth of micrometer resolution, stacking single ceramic sheets to reduce needed length of coil winding and dimension of the coil itself [3], and an ability to operate in high frequency area predetermines using LTCC as a basic substrate for planar inductor coil. High reliability and stability of realised structures and excellent electrical characteristics [4, 5] predetermine LTCC for use in sensor's applications. Reducing total size of sensing device and increasing reliability of sensing module can be achieved

\footnotetext{
${ }^{1}$ Department of Technologies in Electronics

Faculty of Electrical Engineering and Informatics

Technical University of Kosice

Letna 9, 04200 Kosice, Slovak Republic

*Corresponding author, e-mail: samuel.zuk@tuke.sk
} 
by integrating sensor's driving electronic system together with planar coil into multilayer LTCC module.

Inductive sensing system is based on penetration of electromagnetic field generated by sensor's coil into target. Own electromagnetic fields that react with the sensor's coil field are generated by currents induced in target material, so minimum considerations on target size and thickness are required $[6,7]$. With raising driven frequency minimum thickness of sensed target goes down. Reducing planar coil's dimensions and working at higher frequencies ( $\mathrm{MHz}$ range) sensing even small and thin conductive targets can be achieved [8].

Principle of inductive sensors' operation allows using these sensors only for metal - based objects sensing. In dependence of sensing coil's area, limited sensing proximity range can be achieved. Inductive sensors consist of sensing coil and driving oscillating circuit.

For manufacturing industry, there is huge potential for inductive sensors especially for process control. When requirements for measuring proximity ranges in few millimetres distance [9], standardly used inductive proximity sensor requires a lot of space for mounting. There had been presented a lot of inductive proximity sensors based on $\operatorname{LTCC}[1,3,10]$. The dimensions of mentioned sensors are relatively small (from $4.7 \times 2.4 \mathrm{~mm}$ up to $15.7 \times 8.7 \mathrm{~mm}$ ), but these sensors provides the best sensitivity at micrometre ranges and can be used to measure ranges only up to $4 \mathrm{~mm}$. Because of very small area and high resistance of sensing coils (up to $120 \Omega$ ), these sensors achieves small quality factor (ratio of stored vs. dissipated energy per unit time). Complex and expensive, but reliable and stable electronic circuits are used to drive these coils.

Design of a compact, multilayer proximity inductive sensor element with small dimensions, but long range sensing distance and universal electronic module for driving inductive sensors and processing measured values is presented in this paper.

\section{Planar coil design}

Accepting advantages and disadvantages of different coil shapes [11] and also of technological possibilities of LTCC technology for given coil's shape, geometrical parameters of developed planar inductive sensor were determined. To minimize loses and to obtain the best performance of inductive sensor for our application, coil with circle shape shows as the ideal option.

\subsection{Coil's parameter calculation}

To calculate inductance $L$ of single-layer planar coil of different shapes, Eq. (1) [12] is used:

$$
L=K_{1} \mu_{0} \frac{n^{2} d_{A V G} c_{1}}{1+K_{2} \rho}\left(\ln \left(\frac{c_{2}}{\rho}\right)+c_{3} \rho+c_{4} \rho^{2}\right),
$$

where $\mu_{0}$ is the permeability of free space, $n$ is the number of planar coil's turns, $K_{1}, K_{2}$ and $c_{1}$ to $c_{4}$ are constants geometry dependent on the shape of the inductor (for circle shape, $\mathrm{c}_{1}=1$, $\left.\mathrm{c}_{2}=2.46, \mathrm{c}_{3}=0, \mathrm{c}_{4}=0.2\right), \rho$ is the coil's fill ratio and is equal to Eq. (2) [12]:

$$
\rho=\frac{d_{O U T}-d_{I N}}{d_{O U T}+d_{I N}}
$$

where $d_{I N}$ and $d_{O U T}$ is the inner and outer diameter of the coil, respectively, and $d_{A V G}$ is the average diameter of the turns equal to Eq. (3) [12]:

$$
d_{A V G}=\frac{d_{I N}+d_{O U T}}{2}
$$

Complete calculated parameters of developed coil are listed in Table 1.

Table 1 Complete calculated parameters of developed coil.

\begin{tabular}{ll}
\hline Parameter & Value \\
\hline Outer diameter of coil & $15.82 \mathrm{~mm}$ \\
Inner diameter of coil & $7.82 \mathrm{~mm}$ \\
Geometric mean diameter & 0.338 \\
Number of layers & 6 \\
Turns per layer & 8 \\
Trace width & $0.25 \mathrm{~mm}$ \\
Spacing between traces & $0.25 \mathrm{~mm}$ \\
Conductor paste thickness & $0.02 \mathrm{~mm}$ \\
Self-inductance per layer & $0.954 \mu \mathrm{H}$ \\
Total inductance & $22.556 \mu \mathrm{H}$ \\
AC resistance & $8.807 \Omega$ \\
DC resistance & $6.107 \Omega$ \\
Coil length per layer & $297.069 \mathrm{~mm}$ \\
Coil total length & $1782.414 \mathrm{~mm}$ \\
Spacing between layers & $0.2 \mathrm{~mm}$ \\
Coil fill ratio & 0.494 \\
Skin depth & 0.026 \\
Self-resonant frequency & $14.593 \mathrm{MHz}$ \\
Resonance impedance & $25611.29 \Omega$ \\
\hline
\end{tabular}

\subsection{Coil's realization and fabrication}

Sample prototype coil with parameters listed in Table 1 was fabricated using DuPont 6145 silver co-fireable conductor paste screen printed on DuPont GreenTape ${ }^{\mathrm{TM}}$ 951PX ceramic sheets with $254 \mu \mathrm{m}$ thickness. After isostatic lamination (water temperature of $70^{\circ} \mathrm{C}$, pressure of $20.68 \mathrm{MPa}$ ) for 10 minutes, coil was co-fired using recommended [13] 3.5 hours co-fire profile with $850^{\circ} \mathrm{C}$ maximum temperature for 20 minutes. Sensor's coil has circle shape with $15.82 \mathrm{~mm}$ diameter, $250 \mu \mathrm{m}$ conductor's width and spacing between conductors and consist of six layers. Printing of coil's conductor with even small dimensions was not affected by “moiré pattern". Top side's topology of the developed 
planar coil as one part of 6 layers structure design is presented in Fig. 1. The whole topology of developed coil is shown in Fig. 2.

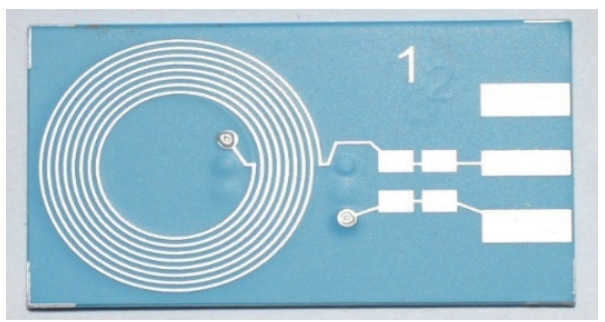

Fig. 1 Developed coil for use in inductive proximity sensor.

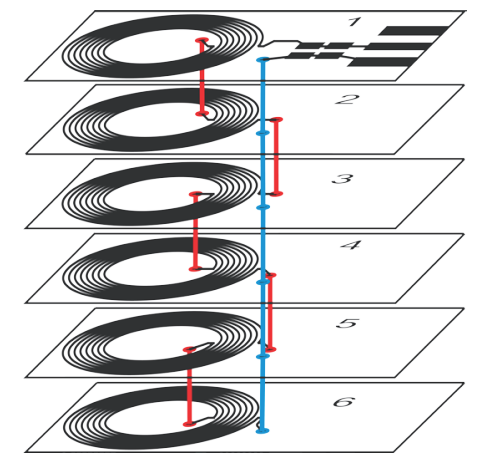

Fig. 2 Internal structure of developed six-layers planar coil (red color - vias connecting two layers, blue color - vias via all six layers).

\subsection{Coil's characterisation}

After proper final inspection, electrical parameters characterisation of developed coil was performed. We decided to use SMA connector to limit parasitical effects of connection to measurement device. There had not been placed additional capacitor on sensing coil during measurement process. Inductance of coil in the frequency range from $300 \mathrm{kHz}$ to $15 \mathrm{MHz}$ was measured by vector network analyser (VNA) N5231A by Keysight Technologies (Fig. 3). Fluctuations in Fig. 3 are caused by measurement step of used VNA. At frequencies higher than $14 \mathrm{MHz}$ parasitic effect of capacitance given by planar structure of coil appears that limits the use of this coil at higher frequencies. Optimizing coil's shape to minimize resistance causing loses and to obtain best performance, developed coil reaches the quality factor of 3 , that is a good value for sixlayer planar coil.

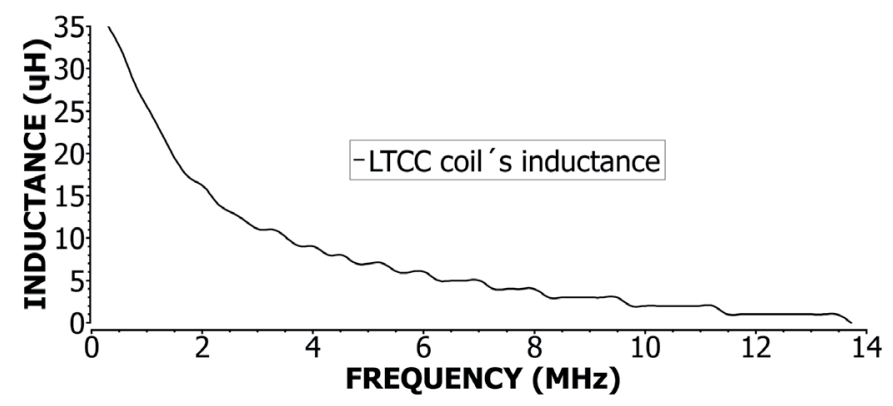

Fig. 3 Developed planar coil's inductance in dependence of driving frequency.

\section{Design of driving electronic module for planar coil}

Inductive sensor for its operation requires driving circuit. Based on performance of driving circuit, behaviour of whole sensing system can be determined. Proper considerations should be taken for choosing appropriate driving circuit to obtain stable and sensitive inductive sensor.

There are several possibilities to drive an inductive proximity sensor, every with its advantages and disadvantages [14]:

- crystal or oscillator of fixed value. Using crystal or oscillator of fixed value brings advantages of simple circuit design and frequency and temperature stability. To reach defined oscillation frequency, an inductor or capacitor tuning is required. When another oscillator frequency is needed, new driving electronic circuit must be build. Another electronic system (e.g. microcontroller) is needed for processing measured values.

- Collpits, Clapp or Hartley oscillators. These types of oscillators are known for wide frequency range and frequency stability [15].

- demodulation circuits, such as frequency modulation (FM), amplitude modulation, phase detection and balanced bridge [9]. High resolution, linearity, high frequency response and low noise are the main advantages of using FM.

- inductance to digital converters (LDC's.).

Inductance to digital converter was considered as an ideal option for purpose of driving a developed coil.

\subsection{LDC based driving electronic module}

Inductance to digital converter is the device that simultaneously measures resonant frequency and impedance of connected parallel LC resonant circuit. Oscillation amplitude of resonant circuit is regulated to a constant level in a closed-loop configuration, and by monitoring power injected into resonant circuit, parallel resistance $R_{P}$ of the coil is determined. By measuring oscillation frequency of resonant circuit, inductance of resonant circuit is determined. LDC's are available in a wide range of mounting packages and configurations [16]:

- 1/2/4 input channels,

- $3,3 \mathrm{~V} / 5 \mathrm{~V}$ operation voltage (analog + digital),

- I2C/SPI interface,

- 12/24/28 bits inductance resolution,

- $8 / 16$ bits parallel resonance impedance resolution,

- $1.8 \mathrm{~V}$ up to $4 \mathrm{~V}$ oscillation amplitude,

- $1 \mathrm{kHz}$ to $10 \mathrm{MHz} / 5 \mathrm{kHz}$ to $5 \mathrm{MHz} / 300 \mathrm{kHz}$ to $19 \mathrm{MHz}$ sensor frequency,

- parallel resistance range from $798 \Omega$ to $3.93 \mathrm{M} \Omega$,

- implemented programmable comparator,

- catalog or automotive rating. 
With minimum external components required, LDC's provides a reasonable solution for inductive sensing from the view of reliability and all-in-one chip solution. For our proximity sensing application, Texas Instruments LDC1000 inductance to digital converter was chosen. Detail specifications of this device are listed in Table 2. Functional block diagram and typical application of LDC1000 inductance to digital converter are presented in Fig. 4 and Fig. 5, respectively. ATmega328 - based microcontroller circuit is used for processing of measured values and communication with computer. To allow using developed driving system with a wide range of different sensing coils, simple program for calibration before every start of sensing application was written. For proper operation some of the LDC1000's configurations registers must be especially configured.

Table 2 Texas Instruments LDC1000 Inductance to Digital converter specifications [17]

\begin{tabular}{ll}
\hline Parameter & Value \\
\hline Number of channels & 1 \\
Supply voltage (analog, digital) & $5 \mathrm{~V}$ \\
Interface & $\mathrm{SPI}$ \\
Inductance resolutions & $24 \mathrm{bits}$ \\
Oscillation amplitude & $1 \mathrm{~V}-4 \mathrm{~V}$ \\
Sensor frequency & $5 \mathrm{kHz}-5 \mathrm{MHz}$ \\
Parallel LC circuit resonance range & $798 \Omega-3.93 \mathrm{M} \Omega$ \\
Parallel resonance impedance resolution & $16 \mathrm{bits}$ \\
Rating & automotive \\
\hline
\end{tabular}

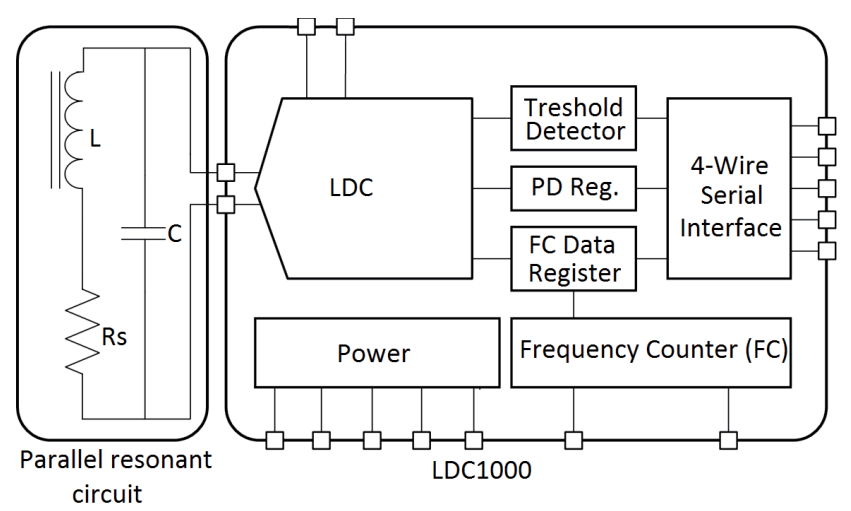

Fig. 4 Functional block diagram of LDC1000 [17].

\subsection{LDC1000 inductance calculations}

There are two values that can be read from LDC1000 using SPI communication - proximity data (PD) and frequency data (FD). To calculate inductance $L$ of the coil, Eq. (4) [17] is being used:

$$
L=\frac{1}{C \cdot\left(2 \cdot \pi \cdot f_{\text {sensor }}\right)^{2}},
$$

where $C$ is the parallel capacitance of the resonator and $f_{\text {sensor }}$ is the sensor frequency equal to Eq. (5) [17]:

$$
f_{\text {sensor }}=\frac{1}{3} \cdot \frac{f_{\text {ext }}}{f_{\text {count }}} \cdot t_{\text {res }}
$$

where $f_{\text {ext }}$ is the external clock's frequency, $f_{\text {count }}$ is obtained FD and $t_{r e s}$ is the programmed response time.

Equation (6) [17] is used to calculate resonance impedance $R_{P}$ of parallel resonant circuit from obtained PD:

$$
R_{P}=\frac{R_{P_{-}} M A X \cdot R_{P_{-}} M I N}{R_{P_{-}} M I N \cdot\left(1-\frac{P D}{2^{15}}\right)+R_{P_{-}} M A X \cdot \frac{P D}{2^{15}}},
$$

where $R_{P_{-}} M A X$ and $R_{P_{-}} M I N$ are programmed maximum and minimum $R_{P}$ that LDC1000 must measure, respectively. These parameters configure the input dynamic range of this device.

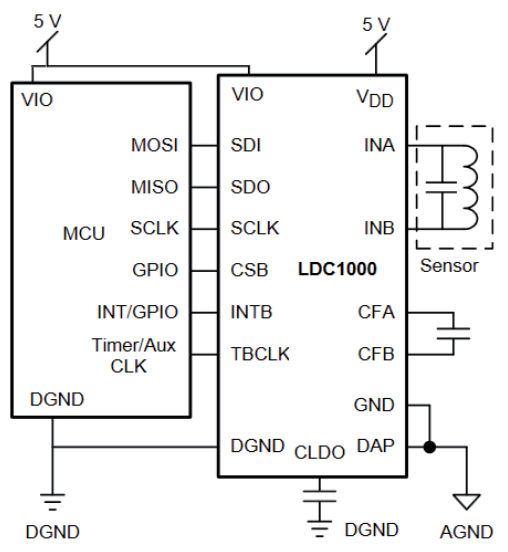

Fig. 5 LDC1000's typical application [17].

\subsection{Results and discussion}

Complete developed LTCC inductive proximity sensor is presented in the Fig. 6. Sensing coil and electronic module are connected using SMA cable.

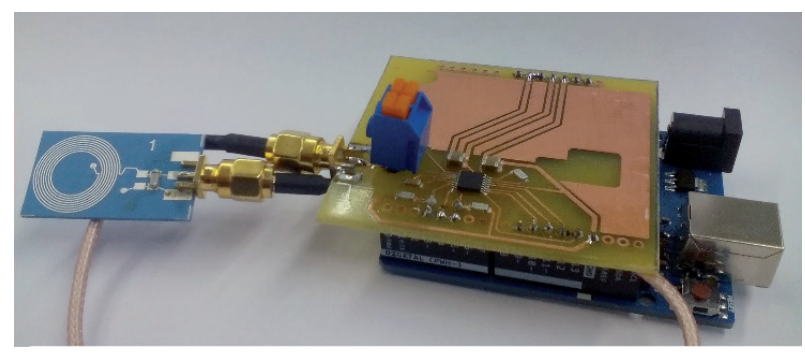

Fig. 6 Complete developed LTCC - based inductive proximity sensor.

Measurements of axial proximity according to Fig. 7 were done to characterize developed inductive proximity system. To obtain comparable results according of inductive proximity sensing requirements [16], these measurements were done with three targets of different material, but thickness of every material was at least $3 \mathrm{~mm}$ and the area of used targets was at least twice in compare of sensing coil's area. 


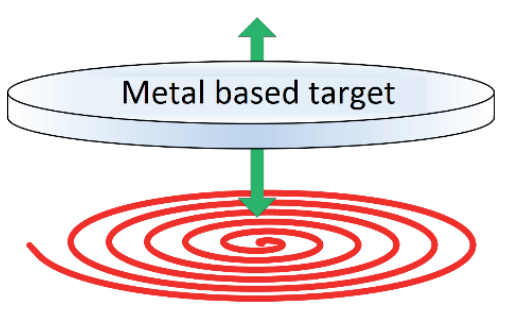

Fig. 7 Principle of inductive proximity sensing measurement. [16]

Measured values of PD and FD depending on distance from target are presented at Fig. 8 and Fig. 9, respectively. Calculated values of parallel resistance $R_{P}$ and inductance $L$ of developed coil are presented at Fig. 10 and Fig. 11, respectively. These measurements were done with three different target materials aluminium, solder material and copper.

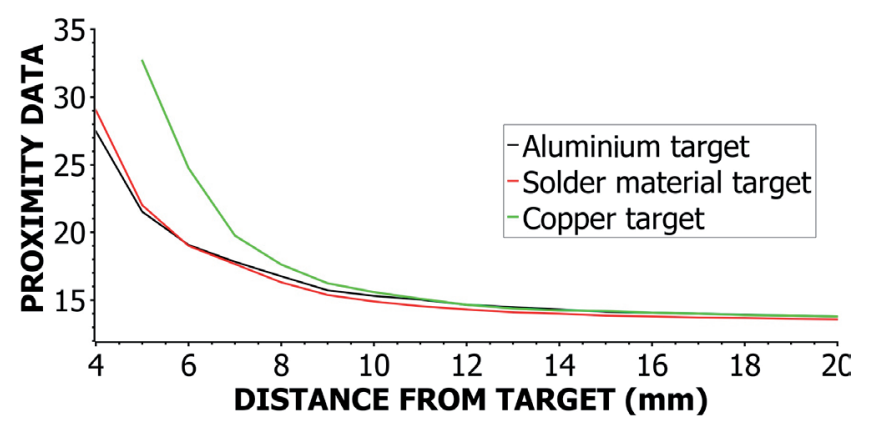

Fig. 8 Measured PD (in thousands) in dependence of distance from target for three different targets.

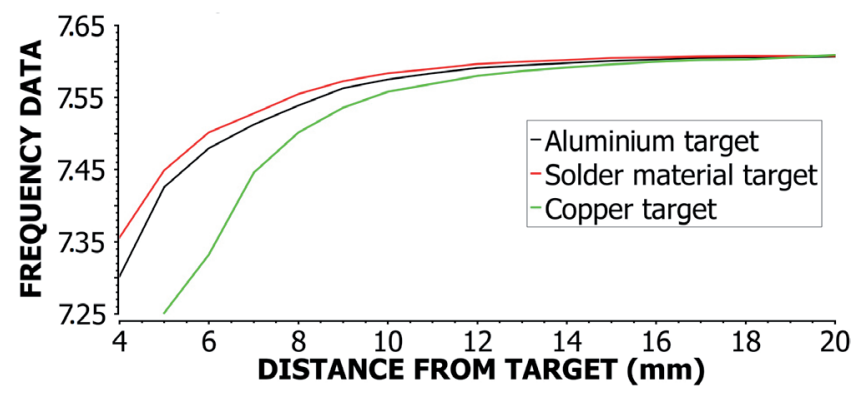

Fig. 9 Measured FD (in thousands) in dependence of distance from target for three different targets.

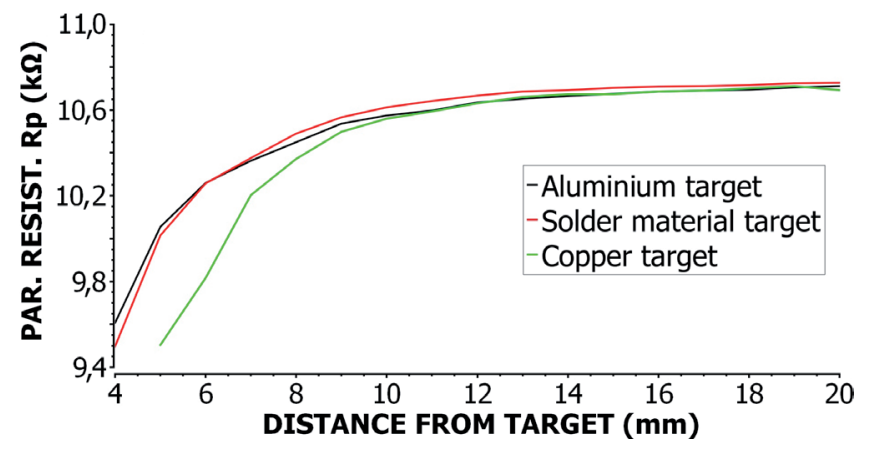

Fig. 10 Calculated parallel resistance $R_{p}$ of developed coil in dependence of distance from the target for different targets.

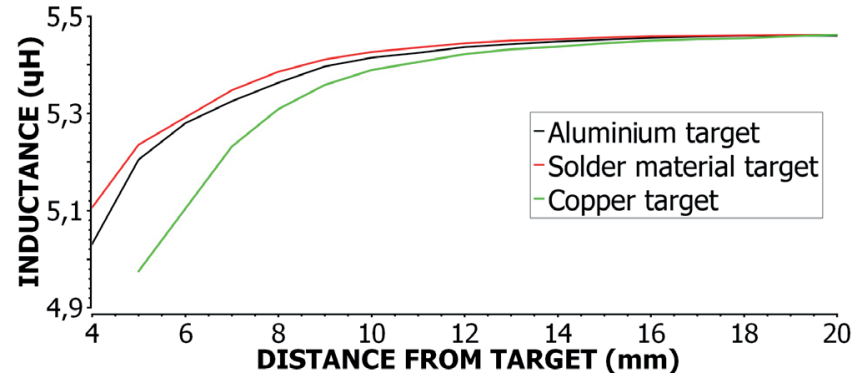

Fig. 11 Calculated inductance L of developed coil in dependence of distance from target for different targets.

At Fig. 8 to Fig. 11 it can be seen, that for distances from $4 \mathrm{~mm}$ to about $10 \mathrm{~mm}$ developed inductive proximity sensior is able to recognize targets from different materials. After the distance of $10 \mathrm{~mm}$ is overreached, inductive sensing system is only able recognize presence of metal based target.

Sensor shows the best performance for copper material target. Targets from aluminium and solder material show very similar performance. These differences are caused by different permeability value of used target materials.

The best sensitivity of planar inductive sensor is in the distance range from $4 \mathrm{~mm}$ to $7 \mathrm{~mm}$. For the measuring distances under $3 \mathrm{~mm}$, sensor must be recalibrated. Measurements showed instability of LDC's output values when measuring proximity range. This instability was caused by improper placement of LDC's driving circuit placed on microcontroller board. To improve stability, driving circuit should be placed directly to sensing coil without necessary long traces or even cables. Using 28-bits driving circuit instead of 24-bits even better sensitivity can be obtained.

Chosen LDC-based electronic system showed electrical characteristics comparable with much complex and far more expensive circuits required for driving inductive proximity sensors. Based on combination of used LTCC technology and allin-one driving chip solution, integration of both components at one substrate can be done to obtain highly reliable, parametric stable and price reasonable inductive sensor.

Developed planar inductive sensor can be used for applications, where copper material target has to be detected. In these sensing applications, recommended proximity sensing distance is 4 to $7 \mathrm{~mm}$.

\section{Conclusion}

In this paper, compact inductive proximity sensing system based on LTCC was introduced and characterized. Using six-layer planar coil with $15.82 \mathrm{~mm}$ diameter we were able to measure up to $20 \mathrm{~mm}$ proximity distance of target.

Inductance to digital converter was confirmed as a reasonable solution for driving inductive sensor. This type driver and planar inductive sensor combination showed performance comparable with much more complex and expensive driving solution. Integrating LDC converter directly at sensing coil 
substrate, a very stable, small dimension and highly reliable planar inductive sensor can be achieved.

Measurements showed that same physical dimensions targets, but of different materials do not behave the same for inductive sensor. Because of that, introduced inductive sensor can be used for target material characterization in limited proximity distance range. The best sensitivity of presented inductive sensor was measured for distances up to $10 \mathrm{~mm}$, repeatability measurement was verified for target distance from 4 to $7 \mathrm{~mm}$.

Developed inductive proximity sensing system will be used for education purposes in sensor's field. Because of ability to drive a coil in a wide frequency range, used driving electronic system will be the base for our future work.

\section{Acknowledgement}

"Paper is the result of the Project implementation: University Science Park TECHNICOM for Innovation Applications Supported by Knowledge Technology, ITMS: 26220220182, supported by the Research \& Development Operational Programme funded by the ERDF." We support research activities in Slovakia. This project is being co-financed by the EU.

"This work was supported by the Slovak Research and Development Agency under the contract No. APVV-14-0085: Development of New Generation Joints of Power Electronics Using Nonstandard Sn-Based Alloys".

\section{References}

[1] Bartsch, H., Geiling, T., Müller, J. "A LTCC low-loss inductive proximity sensor for harsh environments." Sensors and Actuators A: Physical. 175, pp. 28-34. 2012. DOI: 10.1016/j.sna.2011.12.015

[2] Lai, Y. "Eddy current displacement sensor with LTCC technology." PhD Thesis, Albert-Ludwigs Universität Freiburg im Breisgau, 2005.

[3] Gongola-Rubio, M., Sola-Laguna, L., Smith, M., Santiago-Aviles, J. J. "LTCC technology multilayer eddy current proximity sensor for harsh environments." In: Proceedings of the 32nd International Symposium on Microelectronics, IMAPS, Vol. 99, pp. 26-28.

[4] Gongora-Rubio, M. R., Espinoza-Vallejos, P., Sola-Laguna, L., Santiago-Aviles, J. J. "Overview of low temperature co-fired ceramics tape technology for meso-system technology (MsST)." Sensors and Actuators A: Physical. 89(3), pp. 222-241. 2001. DOI: $10.1016 / \mathrm{S} 0924-4247(00) 00554-9$

[5] A. Pietrikova, "Potentiality of LTCC for sensor applications." $24^{\text {th }}$ International Spring Seminar on Electronics Technology: Concurrent Engineering in Electronic Packaging. Calimanesti-Caciulata, May 05-09, 2001, pp. 112-114. DOI: 10.1109/ISSE.2001.931025

[6] Lugli, A. B., Rodrigues, R. B., Santos, M. M. D. "A new method of planar inductive sensor for industrial application." Industrial Electronics Society, IECON 2013 - 39th Annual Conference of the IEEE, Vienna, Austria, Nov. 10-13, 2013, pp. 3858-3863. DOI: 10.1109/IECON.2013.6699751

[7] Wilson, J. S. "Sensor Technology Handbook." Elsevier Inc., 2005.

[8] Wang, H., Li, W., Feng, Z. "Noncontact Thickness Measurement of Metal Films Using Eddy-Current Sensors Immune to Distance Variation." IEEE Transactions on Instrumentation and Measurement. 64(9), pp. 2557-2564. 2015. DOI: 10.1109/TIM.2015.2406053
[9] Wang, H., Liu, Y., Li, W., Feng, Z. "Design of ultrastable and high resolution eddy-current displacement sensor system." In: IECON 2014 - 40th Annual Conference of the IEEE Industrial Electronics Society. Dallas, TX, USA, Oct. 29-Nov. 1, 2014, pp. 2333-2339. DOI: 10.1109/IECON.2014.7048828

[10] Roach, S. D. "Designing and building an eddy current position sensor." Sensors - the Journal of Applied Sensing Technology. 15(9), pp. 56-74. 1998.

[11] Misron, N., Ying, L. Q., Firdaus, R. N., Abdullah, N., Mailah, N. F., Wakiwaka, H. "Effect of inductive coil shape on sensing performance of linear displacement sensor using thin inductive coil and pattern guide." Sensors. 11(11), pp. 10522-10533. 2011. DOI: 10.3390/s111110522

[12] Mohan, S. S., del Mar Hershenson, M., Boyd, S. P., Lee, T. H. "Simple accurate expressions for planar spiral inductances." IEEE Journal of Solid-state Circuits. 34(10), pp. 1419-1424. 1999. DOI: 10.1109/4.792620

[13] DuPont "GreenTape Design Layout Guidelines." 2009.

[14] Zuk, S. "Inductive and capacitive sensors based on film technology." In: SCYR 2016 - Proceedings of 16th Scientific Conference of Young Researchers, TU, Košice, May 2016, pp. 150-153.

[15] Jeranče, N., Bednar, N., Stojanović, G. "An Ink-Jet Printed Eddy Current Position Sensor." Sensors. 13(4), pp. 5205-5219. 2013. DOI: $10.3390 / \mathrm{s} 130405205$

[16] Texas Instruments "LDC sensor design." 2015.

[17] Texas Instruments "LDC1000 5V, High Resolution, Inductance to Digital Converter for Inductive Sensing Applications." 2015. 